\title{
A Robust Scheme for Coherency Detection in Power Systems
}

\author{
Gideon Adom-Bamfi, Emmanuel Asuming Frimpong \\ Department of Electrical and Electronic Engineering, Kwame Nkrumah University of Science and Technology, Kumasi, Ghana
}

\section{ARTICLE INFORMATION}

Received: May 6, 2021

Revised: July 24, 2021

Available online: July 28, 2021

\section{KEYWORDS}

coherence identification, dynamic time

warping, speed deviation, phasor measurement unit, dissimilarity matrix

\section{CORRESPONDENCE}

Phone: +233 (0) 248737456

E-mail: gadom-bamfi.coe@knust.edu.gh

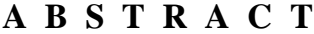

This paper presents an approach for online generator coherency identification based on windowed dynamic time warping (DTW). Generator rotor speed deviations measured by phasor measurement units (PMUs) are used as input data to compute a DTW dissimilarity matrix. Using the dissimilarity matrix together with Agglomerative Hierarchical Clustering (AHC) and Hubert-Levin index (C-index), generators are optimally grouped into coherent clusters. In addition to the clustering of generators, an index for characterizing the transmission delay of a Wide Area Measurement System (WAMS) is presented. A data delay factor that can indicate whether there is an inconsistent PMU data transmission delay is also proposed. The coherency identification technique and indices were tested using simulations carried out on the IEEE 39-bus system. The test results indicate that the proposed scheme accurately clusters generators into coherent groups. The suggested indices were also found to be valid.

\section{INTRODUCTION}

There is a huge demand for electric power in recent times due to growth in population, rise in industrialization, and rapid modernization. Most often, the rise in demand is not accompanied by a commensurate expansion of generation and transmission. This leads to power systems being operated with reduced stability margins. Power systems become stressed under such circumstances.

A stressed power system, when subjected to small signal disturbances like load changes and switching on or off of compensators, may remain stable. However, in the presence of large disturbances such as the tripping of transmission lines due to faults, such a system may lose its stability. Instability of a system could lead to cascading system failures, which could cause equipment damage, pose safety hazards to personnel, contribute to cascading outages, and shutdown of large areas or entire power systems [1].

When a stressed power system is subject to large disturbances that could result in instability, it is necessary to perform emergency control to prevent cascading failures and the collapse of the entire network [2]. The first line of control actions is the engagement of conventional protection systems and controls, e.g., protective relays. However, when the conventional protection and control systems fail to keep the system within stability margins, other actions should be taken. One such control action is Intentional Controlled Islanding (ICI) [3]. ICI is the splitting of a power system into sustainable and stable subsystems called islands. ICI is often used as a last resort to prevent a blackout. The objective of ICI is to create islands by choosing an optimal set of lines to disconnect while minimizing generation-load imbalance, maintaining voltage stability, ensuring coherency of generators, and restraining out-of-step oscillations. The stability of each island depends on the coherency of generators inside the islands. Generators are said to be coherent if they have similar angular trajectories after a fault [3].

For ICI to be effective, correct, and adaptive, online identification of generators' coherency are essential. Moreover, the coherency between groups of generators can change over time, due to changing network topology and operating conditions, thus necessitating real-time coherency identification $[4,5]$. Online measurement-based coherency determination is made feasible due to the increasing deployment of phasor measurement units (PMU) in wide-area measurement systems (WAMS). PMUs can provide synchronized measurements of voltage and current phasors, allowing system observation in real-time.

There are several works on online identification of coherency. In [6], coherent generator groups were identified using discrete Fourier transform (DFT) analysis of internal voltage phasors of generators. The internal voltage phasors were estimated using voltage and current phasors measured by PMUs installed at the 
generator terminals. The approach in [7] also combines generator speed with Fourier analysis to determine coherent generator groups. However, the identification of generator coherency using Fourier transform-based approaches assumes linearity and stationary nature of input data, which does not hold for inter-area oscillations. The works presented in [1], [8], and [9] used Artificial Neural Network (ANN)-based approaches to determine coherent generator groups. ANN-based approaches need extensive offline training which is daunting for large interconnected networks. A correlation coefficient-based method for identifying coherent groups of generators is also proposed in [10]. The method uses a threshold to identify the correct number of groups, which may vary for different operating conditions and fault locations. The threshold requires expert system knowledge which makes practical implementation of the approach challenging.

The methods in [11] and [12] used bioinformatics clustering technique and K-means clustering technique respectively, for the identification of coherent groups. However, both methods require a pre-specification of the number of clusters, which does not make them adaptive. The principal component analysis (PCA) method proposed in [13] employ generator speed and bus voltage angle as input parameter. However, it requires prior information of system dynamic characteristics, which is difficult to obtain. A pattern reorganization technique called independent component analysis (ICA) is used for coherency identification in [14], while in [15], a continuous wavelet transform (CWT) is relied upon.

All the works above assume that data received at control centers from PMUs are time-synchronized, with no data loss. However, there is a possibility of partial data loss or delayed data transmission due to loss of communication from a PMU or latency in a communication channel. Besides, there could be a loss of GPS signal to a PMU [16]. The right identification of coherent generator groups hinges on timely, accurate and synchronized reception of signals from PMUs. As such, if there should be loss or inconsistent delay of data from any of the PMUs, generators could be placed in wrong clusters or classified as singletons. Thus, there is a need for a scheme that rightly determines coherent generator groups in case of data loss or inconsistent delay of data. This need is yet to be addressed.

This work presents a coherency identification scheme that addresses the identified deficiencies in existing techniques. The proposed technique identifies coherent clusters correctly in the event of partial loss of data or inconsistent delay of signals from PMUs. The scheme also identifies coherent clusters correctly when PMU signals are time-synchronized and experience consistent delays. It utilizes the windowed dynamic time warping (DTW) distance [17] as a measure of generator coherency. The DTW distance is used as an input to an agglomerative hierarchical clustering (AHC) algorithm [18] which is used in tandem with the Hubert-Levin index (C-index) [19] to optimally identify coherent generator groups. The input parameters required are rotor speed deviations of the individual generators within a system. The contributions of this work are summarized as follows: (1) Development of a technique for identification of coherent generator clusters based on windowed DTW. (2) Proposition of data delay factor, which is a flag that indicates whether there is an inconsistent data delay or loss, and the PMU signal experiencing such delay. (3) Use of windowed-DTW distance as an index to characterize the PMU data transmission delay of a given wide area measurement system (WAMS).

The rest of the paper is organized as follows: The wide-area measurement system and associated communication challenges are presented in Section 2. Section 3 discusses the concept of generator coherency. In Section 4, Euclidean distance and dynamic time warping distance are compared as similarity metrics. The AHC algorithm as a clustering tool and the $\mathrm{C}$-index as clustering validation index are presented in Section 5 . The proposed coherency identification scheme is presented in Section 6. Test results are presented and discussed in Section 7. Conclusions drawn are highlighted in Section 8.

\section{WIDE AREA MEASUREMENT SYSTEMS}

After many major blackouts in the past, many technologies have been developed and implemented to supervise, monitor, and control power systems during disturbances and sustain their operations. One such technology is the Wide Area Measurement System (WAMS). A WAMS is a system in which phasor measurements are collected from various locations in an electrical grid. The measurements are then transmitted to a central location where they are used for system monitoring and control [20].

\section{WAMS Components}

The basic components of WAMS are phasor measurement units (PMUs), phasor data concentrators (PDCs), and communication networks. Figure 1 shows a block diagram of WAMS. The first basic component of WAMS is the PMU. It is used to measure voltage and current waveforms that are synchronized with clocking signals obtained continuously from a global positioning system (GPS) [21]. Some PMUs can record angles and speeds of synchronous generators [22].

Synchronized measurements offer reliable data for power system analysis, as signals are aligned to common time references, are time-stamped, accurate, and filtered from electrical noise [23].

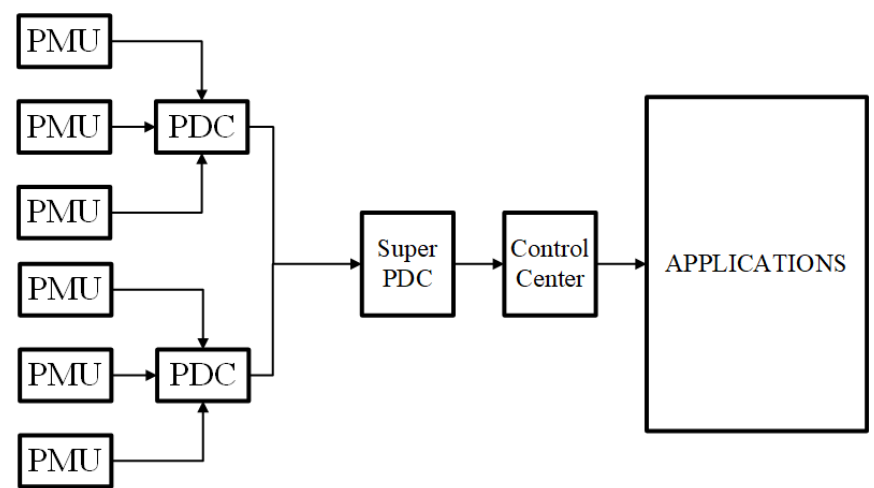

Figure 1. Block diagram of WAMS

The next basic component of WAMS is phasor data concentrators (PDCs). PDCs are smart devices that collect data from PMUs and sort them according to GPS time stamps. PMUs' phasor information in IEEE 1344 data format is transmitted either via dedicated lines between specified locations or over a switched 
link to PDCs. Subsequently, PDCs send time-synchronized data to higher level PDCs called Super PDCs.

The data at the super PDCs are then transmitted to control centers. At these centers, application software packages are applied to the data to yield various preventive and corrective actions.

\section{Communication Infrastructure}

The transmission of measured field data is realized through telecommunication channels such as coaxial cable, fiber optic cable and wireless modems. The latency, jitter, and reliability of communication networks in WAMS are vital in ensuring that WAMS are suitable for supporting protection functions [21]. Communication networks must ensure that measurements supplied by WAMS are timeously and accurately received.

\section{Communication Challenges}

In WAMS, data is transmitted from one logical layer to the other through wired or wireless communication channels. The latency associated with various channels brings about transmission delays. PMU devices are distributed over a wide area, covering various locations, and at varying distances from the PDCs. Thus, data from PMUs will have different end-to-end transmission times. The PDCs' function is to synchronize data transmitted from the PMUs. However, in the event of significant channel delay, the time-out (i.e., the time a PDC has to wait for the arrival of the same time-stamped signals) may be exceeded. Hence, data from some PMUs may not be passed to control centers on time. This leads to the issue of incomplete data [20].

\section{GENERATOR COHERENCY}

Generator coherency identification is primarily a similarity matching problem. The goal is to cluster generators into groups based on the similarity of their measured signal trajectories. When a disturbance occurs in a power system, the generators' responses are governed by their inertia and location in the system. Generators that exhibit similarity in their time-domain responses are considered as coherent and can be clustered.

Following a transient disturbance, individual generators or groups of generators tend to oscillate. Their rotor angle swings are dependent on each other and they evolve together with time. This can be expressed as [1]:

$$
\delta_{p}(t)-\delta_{q}(t) \cong K_{p q}(t) \quad 0 \leq t \leq t_{\max }
$$

where $p$ and $q$ are pairs of generators, $K_{p q}(t)$ is a constant whose value may change with time. The value of $K_{p q}(t)$ will be small and nearly constant for a coherent pair, with the generators said to be perfectly coherent when $K_{p q}(t)=0$. A group of generators are said to be coherent if each pair of generators in the group is coherent according to the above definition. For a non-coherent pair, $K_{p q}(t)$ will be large and may also largely vary with time.

Generally, in power system studies, a pair of generators are classified to be coherent when the value of $K_{p q}(t)$ does not exceed $180^{\circ}, 1$ second after a disturbance [24]. Differentiating the terms in Eq. (1) results in Eq. (2) as follows:

$$
\frac{d \delta_{i}(t)}{d t}-\frac{d \delta_{j}(t)}{d t} \cong C_{p q}(t)
$$

From which

$$
\Delta \omega_{i}-\Delta \omega_{j} \cong C_{p q}(t)
$$

where $C_{p q}(t)$ is another constant whose value may also change with time. From (3), it can be concluded that following a disturbance, the difference in speed deviation of coherent generators, like the difference in rotor angles, will be small and nearly constant, while the difference in speed deviation of pairs or group of generators that go out of step will be large and also vary.

Rotor speed deviations of generating units can be obtained with the help of some special PMUs which can record synchronous generators' speeds through theoretical calculation on electrical measurements and key phase pulse signals [1]. Thus, algorithms based on rotor speed deviations can be implemented in the field.

\section{COMPARISON OF EUCLIDEAN DISTANCE AND DYNAMIC TIME WARPING DISTANC}

Several time-domain coherency identification schemes employ the Euclidean distance or its variants such as the Manhattan distance, Maximum distance, Minkowski distance and Mahalanobis distance. The Euclidean distance is a commonly used tool to measure similarity between signals. Euclidean distance can only be computed for vectors of equal length. For two trajectories or time series vectors $T$ and $S$ of equal length $n$, the Euclidean distance $d_{\text {euc }}$ between their $i$ th samples is:

$$
d_{\text {euc }}(T, S)=\sqrt{\sum_{i=1}^{n}\left(T_{i}-S_{i}\right)^{2}}
$$

For any two trajectories, the smaller the Euclidean distance, the more similar they are, and vice versa. For any generator pair, the smaller the Euclidean distance, the more coherent they are.

The Euclidean distance and its variants consider the differences of the samples of two time series at corresponding time points and do not consider the consistency of the changes in time series. Consequently, the Euclidean distance as a measure of similarity is sensitive to signal shifts [17]. For example, if one time series in a pair that is coherent in nature is only slightly delayed or shifted from the other, but otherwise the same, the Euclidean distance between them will be large and thus be misinterpreted to be noncoherent. This is illustrated in Figure 2(a). In the figure, the red and blue curves correspond to two signals, with the blue signal delayed. It can be noticed that the two signals are similar, but the positions of peaks and troughs are not exactly aligned due to the delay of the blue signal. The two time series are not considered to be similar under Euclidean distance, because Euclidean distance can only examine the difference of values of different samples at corresponding time points, without considering the consistency of sample changes in time series. As such, the use of Euclidean distance gives an inaccurate measure of similarity in the practical events of signal delay due to communication channel latency. On the other hand, dynamic time warping (DTW) as a measure of similarity, gives more robustness to similarity computation. By this method, time series of different lengths and speeds can be compared. DTW replaces the one-to-one point comparison, used in Euclidean distance, with a many-to-one or one-to-many comparison as seen in Figure 2(b). The main feature of this 
distance measure is that it allows the recognition of similar trajectories, even if there is a delay or loss of samples. It does this by finding the optimal match between two time series. For time series $T$ and $S$, the DTW distance $d_{d t w}$ between them is given as:

$$
d_{d t w}(T, S)=\min \left\{d_{w}(T, S)\right\}
$$

where $d_{d t w}(T, S)$ is the cost of matching the two time series, computed by summing the local distances between samples of the two time series defined according to the non-linear mapping between the them.

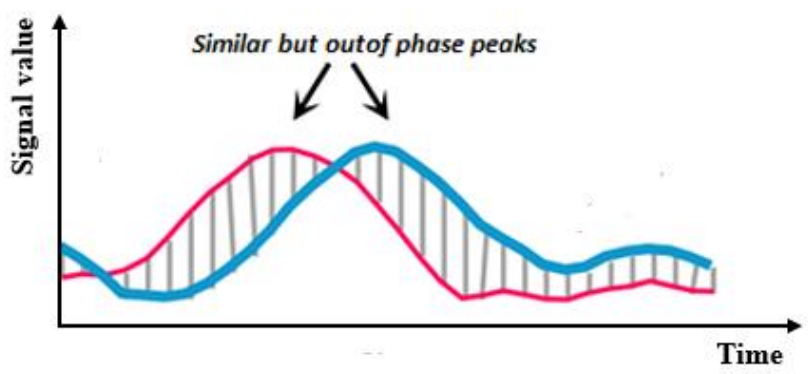

(a)

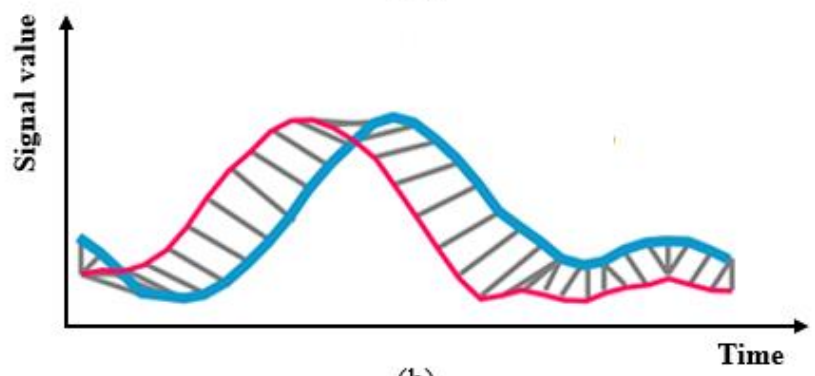

(b)

Figure 2. (a) Euclidean distance used as a measure of similarity, (b) DTW used as a measure of similarity

\section{AGGLOMERATIVE HIERARCHICAL CLUSTERING ALGORITHM AND C-INDEX}

\section{Agglomerative Hierarchical Clustering as a Clustering tool}

The AHC is a type of hierarchical clustering technique used to group objects in a bottom-up manner. The algorithm starts by treating each object as a singleton cluster. Next, the singleton clusters are merged until a desired number of clusters that satisfy stated conditions are formed [18].

The AHC algorithm has three main distance measures. These are: single linkage, complete linkage, and average linkage [18]. Generally, the AHC algorithm with a single linkage is most stable and effective. Thus, the single linkage measure is used for the cluster analysis in this work. The AHC algorithm is also called the nearest neighbor cluster algorithm when the single linkage measure is used for the distance between clusters.

Considering a set of $N$ objects, and seeking to form $K$ clusters using a single linkage, the basic algorithm of the AHC is presented using the flowchart shown as Figure 3. This is further outlined as follows[25]:
Step 1: Specify the desired number of clusters, $K$ from the range $[1, N]$.

Step 2: Form an initial singleton cluster, $G_{i}$ for each object $i$.

Step 3: Find the distances between each pair of clusters and construct the distance (dissimilarity) matrix, D, of size $C \times C$ where $C$ is the number of clusters formed.

Step 4: Merge the clusters with the smallest dissimilarity (say, $G_{p}$ and $G_{q}$ into new cluster, $G_{r}$ and decrease $C$ by 1 .

Step 5: Check the updated number of clusters formed. If this number is greater than the desired number of clusters, $k$, go to Step 3; otherwise, proceed to Step 6.

Step 6: Output the clustering results.
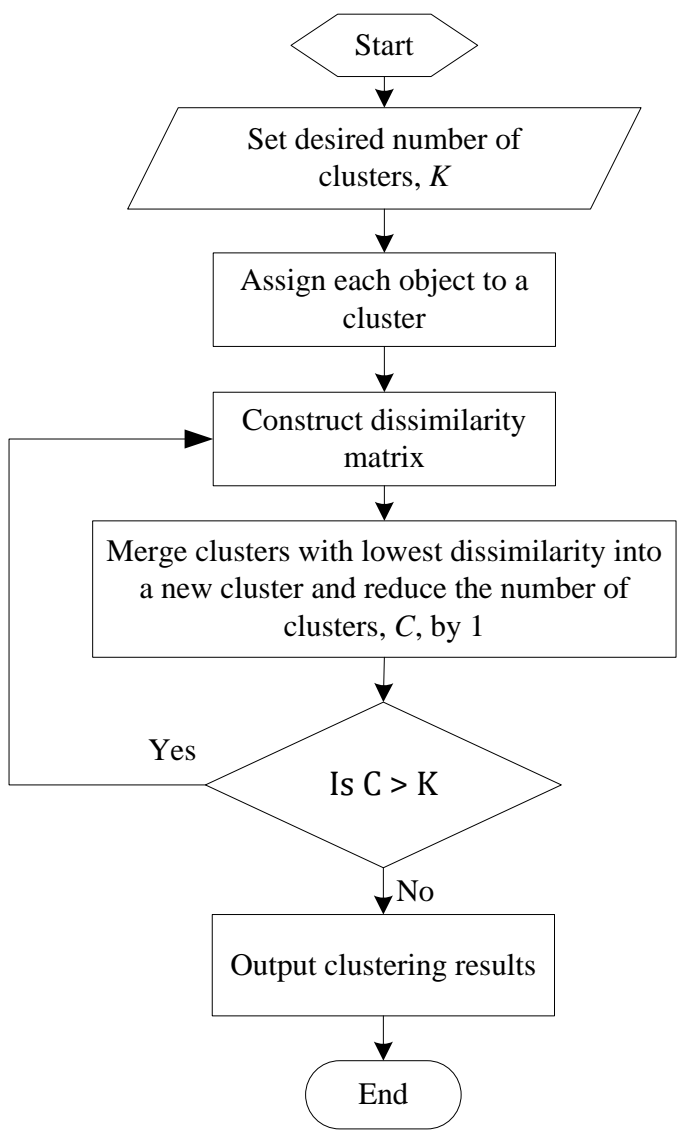

Figure 3. Flowchart of the AHC algorithm

\section{Hubert-Levin Index (C-Index) as a Cluster Evaluation Index}

The Hubert-Levin index (HL or C-index) was used together with the AHC algorithm to find the optimal clustering results. The HL index is a measure of compactness. It takes into account, the within-cluster distances. The index varies between 0 and 1 . A good clustering result corresponds to low values of the index [26]. For a clustering result, the HL-index is given by [26]:

$$
H L=\frac{S_{w}-S_{\min }}{S_{\max }-S_{\min }}
$$

Where $S_{w}$ is the sum of the within-cluster distances; $S_{\min }$ is the sum of the smallest distances considering all pairs of clusters; and $S_{\max }$ is the sum of the greatest distances considering all pairs of clusters. 


\section{GENERATOR COHERENCY IDENTIFICATION BASED ON DYNAMIC TIME WARPING (DTW)}

\section{DTW as a Scheme for Coherency Identification}

Consider two rotor speed deviation trajectories specified as a data matrix as follows:

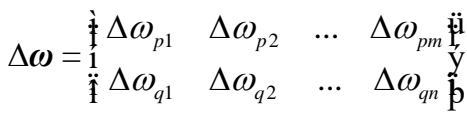

Where $\Delta \omega$ corresponds to the speed deviation matrix for an event, $m$ and $n$ correspond to the number of data points for generators $p$ and $q$ respectively. Normally, for time-synchronized PMU measurements, $m$ and $n$ are equal. Under such a scenario, Euclidean distance or its variant can be used as an accurate measure of coherency.

However, in the event of a relative delay of a trajectory, for instance, by a sample period as a result of transmission channel latency, the following matrix results:

$$
\Delta \boldsymbol{\omega}^{*}=\left\{\begin{array}{llll}
\Delta \omega_{p 1} & \Delta \omega_{p 2} & \ldots & \Delta \omega_{p m} \\
\Delta \omega_{q 0} & \Delta \omega_{q 1} & \ldots & \Delta \omega_{q(n-1)}
\end{array}\right\}
$$

Where $\Delta \omega^{*}$ corresponds to the speed deviation matrix for generators $p$ and $q$ for a sample-period delay in the trajectory of generator $q$.

In (8), it is assumed that there is a delay of PMU signals from generator $q$. The shift in the trajectory means that, even if the two trajectories are similar and thus coherent, the use of Euclidean distance or its variant would indicate otherwise, because, at any point in time, a current sample and a delayed sample of generators $p$ and $q$ respectively will be compared. For example, at the second sample point, speed deviations $\Delta \omega_{p 2}$ and $\Delta \omega_{q 1}$ corresponding to the generators $p$ and $q$ respectively will be compared if the Euclidean distance is used. Also, at the last sample points, speed deviations $\Delta \omega_{p m}$ and $\Delta \omega_{q(n-1)}$ for generators $p$ and $q$ respectively are compared. Consequently, the use of Euclidean distance would give an inaccurate measure.

To overcome the drawbacks associated with the use of Euclidean distance and other point-to-point comparison metrics for coherency identification, DTW is proposed in this work. For the two rotor speed deviation trajectories for $p$ and $q$, estimated over the same period, DTW can be used whether there is a delay or data loss due to PMU data transmission.

To match these two trajectories using DTW, a local cost measure, $d\left(\Delta \omega_{p i}, \Delta \omega_{q j}\right)$, is defined. The local cost refers to the vertical distance between data points $i$ and $j$ of rotor speed deviation trajectories $\Delta \omega_{p}$ and $\Delta \omega_{q}$ respectively. It indicates the similarity between the two data points, and is defined as:

$$
d\left(\Delta \omega_{p i}, \Delta \omega_{q j}\right)=\left|\Delta \omega_{p i}-\Delta \omega_{q j}\right|^{2}
$$

Where $i \in\{1,2, \ldots ., m\}$ and $j \in\{1,2, \ldots \ldots, n\}$.
Typically, $d\left(\Delta \omega_{p i}, \Delta \omega_{q j}\right)$ is small (low cost) if $\Delta \omega_{p i}$ and $\Delta \omega_{q j}$ are similar to each other, otherwise, $d\left(\Delta \omega_{p i}, \Delta \omega_{q j}\right)$ is large (high cost).

A local cost matrix distMatrix $\left(\Delta \omega_{p}, \Delta \omega_{q}\right)$ of size $m$-by- $n$ is constructed by calculating local cost measures of each pair of data points from trajectories $\Delta \omega_{p}$ and $\Delta \omega_{q}$ as follows:

$$
\text { distMatrix }=\left\{\begin{array}{cccc}
d\left(\Delta \omega_{p 1}, \Delta \omega_{q 1}\right) & d\left(\Delta \omega_{p 1}, \Delta \omega_{q 2}\right) & \ldots & d\left(\Delta \omega_{p 1}, \Delta \omega_{q n}\right) \\
d\left(\Delta \omega_{p 2}, \Delta \omega_{q 1}\right) & d\left(\Delta \omega_{p 2}, \Delta \omega_{q 2}\right) & \ldots & d\left(\Delta \omega_{p 2}, \Delta \omega_{q n}\right) \\
\vdots & \vdots & \vdots & \vdots \\
d\left(\Delta \omega_{p m}, \Delta \omega_{q 1}\right) & d\left(\Delta \omega_{p m}, \Delta \omega_{q 2}\right) & \ldots & d\left(\Delta \omega_{p m}, \Delta \omega_{q n}\right)
\end{array}\right\}(10)
$$

Where $1 \leq i \leq m$ and $1 \leq j \leq n$ and entry $d\left(\Delta \omega_{p i}, \Delta \omega_{q j}\right)$, corresponds to the vertical distance of $i$ th point of $\Delta \omega_{p}$ and $j$ th point of $\Delta \omega_{q}$.

To find the best match between the two trajectories, a path through the distMatrix called a warping or alignment path, is found. A warping path, $W$, is a contiguous set of matrix elements that characterizes a mapping between two trajectories by assigning element $\Delta \omega_{p i}$ of $\Delta \omega_{p}$ to element $\Delta \omega_{q j}$ of $\Delta \omega_{q}$. A warping path, $\mathrm{W}$, is defined as:

$$
W=\left\{w_{1}, w_{2}, \ldots, w_{k} \ldots, w_{K}\right\}
$$

where $w_{k}=\left(i_{k}, j_{k}\right) \epsilon[1: m] \times[1: n]$ represents the cell in the $i_{k}$ th row and $j_{k}$ th column of the distance matrix $\operatorname{distMatrix}\left(\Delta \omega_{p}, \Delta \omega_{q}\right)$.

For example, in Figure 4, an optimal warping path for the red and blue signals is shown by the red cells. Given that $w_{k}=\left(i_{k}, j_{k}\right)$ and $w_{k-1}=\left(i_{k}{ }^{\prime}, j_{k}{ }^{\prime}\right)$ with $i_{k}, i_{k}{ }^{\prime} \leq m$ and $j_{k}, j_{k}{ }^{\prime} \leq n$, a valid warping path is subject to the following constraints [27]:

(1) Boundary conditions: It enforces that the first elements, $w_{1}=(1,1)$ and the last elements $w_{K}=(m, n)$ of $\Delta \omega_{p}$ and $\Delta \omega_{q}$ are aligned to each other.

(2) Continuity: This avoids omissions in elements and replications in the alignment of $\Delta \omega_{p}$ and $\Delta \omega_{q}$ with $i_{k}-$ $i_{k}{ }^{\prime} \leq 1$ and $j_{k}-j_{k}{ }^{\prime} \leq 1$.

(3) Monotonicity: This forces the points in the warping path to be monotonically spaced in time such that $i_{k}-i_{k}{ }^{\prime} \geq 0$ and $j_{k}-j_{k}{ }^{\prime} \geq 0$.

It should be noted that when the two trajectories $\Delta \omega_{p}$ and $\Delta \omega_{q}$ are similar and time-synchronized, the warping path is the diagonal of the distance matrix.

The total or global cost $d_{w}\left(\Delta \omega_{p}, \Delta \omega_{q}\right)$ of a warping path $W$ between $\Delta \omega_{p}$ and $\Delta \omega_{q}$ with respect to the local cost measure is defined as:

$$
d_{w}\left(\Delta \omega_{p}, \Delta \omega_{q}\right)=\sqrt{\sum_{k=1}^{K} d\left(\Delta \omega_{p i_{k}}, \Delta \omega_{q j_{k}}\right)}
$$




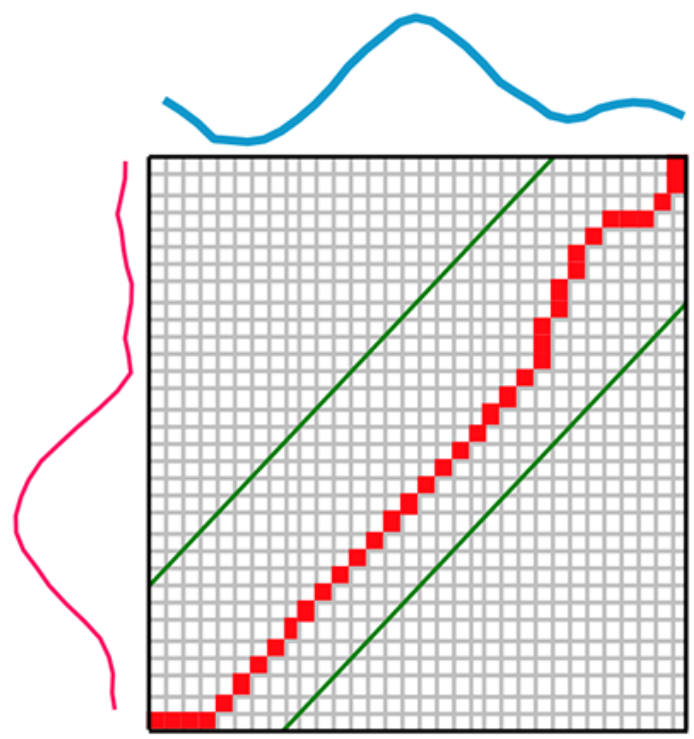

Figure 4. Optimal warping path for two signals

The objective of DTW is to find the distance $d_{d t w}\left(\Delta \omega_{p}, \Delta \omega_{q}\right)$ between two trajectories $\Delta \omega_{p}$ and $\Delta \omega_{q}$, that gives the minimum total cost among all possible warping paths. Mathematically,

$$
d_{d t w}\left(\Delta \omega_{p}, \Delta \omega_{q}\right)=\min \left\{d_{w}\left(\Delta \omega_{p}, \Delta \omega_{q}\right)\right\}
$$

This distance, referred to as the DTW distance, is efficiently computed using dynamic programming [17]. By this method, a cumulative distance matrix, $\gamma$, of the same dimension as the distance matrix, is created to store in the cell $(i, j)$ of $\gamma$, the following value:

$$
\begin{aligned}
& \gamma(i, j)= \\
& \begin{cases}d\left(\Delta \omega_{p i}, \Delta \omega_{q j}\right)+\min \{\gamma(i-1, j-1), \gamma(i-1, j), \gamma(i, j-1)\} & |i-j| \leq \varepsilon \\
\infty & \text { otherwise }\end{cases}
\end{aligned}
$$

Where $d\left(\Delta \omega_{p i}, \Delta \omega_{q j}\right)$ is the local cost measure between the $i$ th and $j$ th samples of $\Delta \omega_{p}$ and $\Delta \omega_{q}$ respectively and $\varepsilon$ is the warping window threshold, defined to overcome the problem of mapping a large number of points of $\Delta \omega_{p}$ to a single point of $\Delta \omega_{q}$ and vice versa. From (14), it implies the cumulative distance $\gamma$ at the $(i, j)$ cell will consider besides the local cost between $\Delta \omega_{p i}$ and $\Delta \omega_{q j}$, the minimum value among adjacent cells at positions: $(i-1, j-$ $1),(i-1, j)$ and $(i, j-1)$. By this method, the last element of $\gamma$ corresponds to the DTW distance. As a special case, when the warping window threshold is zero, the DTW is equal to the Euclidean distance.

The DTW distance is also defined as the cost of matching two trajectories. Thus, if two trajectories are similar and timesynchronized (with consistent delay), the DTW distance will be less than when there are unequal or inconsistent delays of the signals. In such case, the DTW distance, $d_{d t w}\left(\Delta \omega_{p}, \Delta \omega_{q}\right)$ is also equal to the Euclidean distance between the two trajectories. Thus, the Euclidean distance between two trajectories can be seen as a special case of DTW, defined as the case where the two trajectories are time-synchronized.

\section{Proposed Measures of Generator Coherency and Signal Delay}

\section{Coherency Measure}

In this work, the DTW distance, $d_{d t w}\left(\Delta \omega_{p}, \Delta \omega_{q}\right)$ is proposed as a measure of generator coherency. Two generators $p$ and $q$ are coherent if they satisfy the following equation:

$$
d_{d t w}\left(\Delta \omega_{p}, \Delta \omega_{q}\right) \leq \delta
$$

Where $\delta$ is a threshold value. For non-coherent generator pairs or groups, the DTW distance will exceed the threshold value. Setting of the threshold value is subjective and could lead to inaccurate results. The need to explicitly define the thresholds for various groups is avoided by using the AHC algorithm coupled with the C-index in this work.

For coherency identification among a given set of generators in a power system, a matrix referred to as the dissimilarity matrix is computed. This comprises the DTW distances of each pair of speed deviation trajectories of the generators in the set. The dissimilarity matrix for a power system of $N$ number of generators is given by:

$$
d_{d t w}=\left\{\begin{array}{cccc}
d_{d t w}\left(\Delta \omega_{1}, \Delta \omega_{1}\right) & d_{d t w}\left(\Delta \omega_{1}, \Delta \omega_{2}\right) & \ldots & d_{d t w}\left(\Delta \omega_{1}, \Delta \omega_{N}\right) \\
d_{d t w}\left(\Delta \omega_{2}, \Delta \omega_{1}\right) & d_{d t w}\left(\Delta \omega_{2}, \Delta \omega_{2}\right) & \ldots & d_{d t w}\left(\Delta \omega_{2}, \Delta \omega_{N}\right) \\
\vdots & \vdots & \vdots & \vdots \\
d_{d t w}\left(\Delta \omega_{N}, \Delta \omega_{1}\right) & d_{d t w}\left(\Delta \omega_{N}, \Delta \omega_{2}\right) & \ldots & d_{d t w}\left(\Delta \omega_{N}, \Delta \omega_{N}\right)
\end{array}\right\}
$$

The dissimilarity matrix is then normalized to form the matrix $\boldsymbol{d}_{\boldsymbol{d} t \boldsymbol{w}}^{*}$ whose elements are determined according to the equation:

$$
\begin{aligned}
& d_{d t w}^{*}\left(\Delta \omega_{p}, \Delta \omega_{q}\right)= \\
& \frac{d_{d t w}\left(\Delta \omega_{p}, \Delta \omega_{q}\right)-\min _{p, q \in\{1,2, \ldots N\}}\left\{d_{d t w}\left(\Delta \omega_{p}, \Delta \omega_{q}\right)\right\}}{\max _{p, q \in\{1,2, \ldots N\}}\left\{d_{d t w}\left(\Delta \omega_{p}, \Delta \omega_{q}\right)\right\}-\min _{p, q \in\{1,2, \ldots N\}}\left\{d_{d t w}\left(\Delta \omega_{p}, \Delta \omega_{q}\right)\right\}}
\end{aligned}
$$

Where $d_{d t w}^{*}\left(\Delta \omega_{p}, \Delta \omega_{q}\right)$ denotes the normalized value of the DTW distance between the speed deviation trajectories of generators $p$ and $q$ respectively. The normalized DTW dissimilarity matrix is further used in the coherency identification algorithm.

\section{PMU Data Delay Factor}

When there is no data loss or inconsistencies in PMU data transmission delays, the DTW distance between a pair of coherent generators is equal to the Euclidean distance $d_{\text {euc }}\left(\Delta \omega_{p}, \Delta \omega_{q}\right)$ between their speed deviation trajectories. That is:

$$
d_{d t w}\left(\Delta \omega_{p}, \Delta \omega_{q}\right)=d_{e u c}\left(\Delta \omega_{p}, \Delta \omega_{q}\right)=\sqrt{\sum_{i=1}^{m}\left\{d\left(\Delta \omega_{p i}, \Delta \omega_{q i}\right)\right\}^{2}}
$$

To determine the generator whose PMU data is missing or experiencing inconsistent delay with respect to the other generators' signals in a power system, thereby enhancing the coherency identification algorithm performance, an index referred to as data delay factor $d_{d e l}$ is also proposed in this work. The data delay factor is also a measure of the data delay of a given PMU signal. For generators $p$ and $q$, the data delay factor is given 
by the ratio of the DTW distance, $d_{d t w}\left(\Delta \omega_{p}, \Delta \omega_{q}\right)$ and the Euclidean distance $d_{\text {euc }}\left(\Delta \omega_{p}, \Delta \omega_{q}\right)$, which is given as:

$$
d_{d e l}\left(\Delta \omega_{p}, \Delta \omega_{q}\right)=\frac{d_{d t w}\left(\Delta \omega_{p}, \Delta \omega_{q}\right)}{d_{e u c}\left(\Delta \omega_{p}, \Delta \omega_{q}\right)}
$$

For two time-synchronized coherent generators, $d_{d e l}$ has a value of unity, because the DTW distance will be equal to the Euclidean distance. However, for coherent generators with unequal delay times of their PMU data, $d_{d e l}$ is less than one as the Euclidean distance will be greater than the DTW distance. Also, for a noncoherent generator pair, $d_{d e l}$ is approximately unity for both equal and unequal delay times. This is because the Euclidean distance barely deviates from the DTW distance for non-coherent signals [28]. In addition, for perfectly coherent generators with time-synchronized PMU data, $\quad d_{\text {euc }}\left(\Delta \omega_{p}, \Delta \omega_{q}\right)$ and $d_{d t w}\left(\Delta \omega_{p}, \Delta \omega_{q}\right)$ will be zero, giving a divide-by-zero error However, this is a rare scenario, as there would not be perfectly coherent generators in a given time-window.

A delay factor matrix $\boldsymbol{d}_{\boldsymbol{d e l}}$ is formed in which each cell is the ratio of the DTW distance and Euclidean distance for two generators. For a system with $N$ generators, the data delay factor matrix is given as:

$$
d_{d e l}=\left\{\begin{array}{cccc}
d_{d e l}\left(\Delta \omega_{1}, \Delta \omega_{1}\right) & d_{d e l}\left(\Delta \omega_{1}, \Delta \omega_{2}\right) & \ldots & d_{d e l}\left(\Delta \omega_{1}, \Delta \omega_{N}\right) \\
d_{d e l}\left(\Delta \omega_{2}, \Delta \omega_{1}\right) & d_{d e l}\left(\Delta \omega_{2}, \Delta \omega_{2}\right) & \ldots & d_{d e l}\left(\Delta \omega_{2}, \Delta \omega_{N}\right) \\
\vdots & \vdots & \vdots & \vdots \\
d_{d e l}\left(\Delta \omega_{N}, \Delta \omega_{1}\right) & d_{d e l}\left(\Delta \omega_{N}, \Delta \omega_{2}\right) & \ldots & d_{d e l}\left(\Delta \omega_{N}, \Delta \omega_{N}\right)
\end{array}\right\}
$$

\section{DTW-distance-based Coherency Identification Algorithm}

The proposed algorithm for the identification of Coherent Groups (CGs) uses the dissimilarity matrix as the input parameter. After the evaluation of the dissimilarity matrix, the next step is to identify the CGs. The AHC algorithm is adopted to aid the identification of CGs. The AHC is used together with the C-index to determine the optimal clusters, and the optimal number of clusters $k_{\text {opt }}$ from the clustering number search range $\left[k_{\min }, k_{\max }\right] . k_{\min }$ is set to be greater than unity to avoid putting all generators into one cluster. $k_{\max }$ is set not so high to avoid putting generators into singleton clusters. The search range also impacts the computational time of the algorithm. The wider the range, the higher the computational time and vice versa. The flowchart of the coherency identification algorithm is as shown as Figure 5. Its operation is outlined as follows:

Step 1: Obtain speed deviation data from PMUs in the time window, $t=0.05 \mathrm{~s}-0.55 \mathrm{~s}$ after the clearance of a fault.

Step 2: Set the search range $\left[k_{\min }, k_{\max }\right]$ for the number of clusters.

Step 3: Initially assign each generator to a unique cluster, $G_{i}=$ $\{i\}(i=1,2, \ldots \ldots \ldots N)$.

Step 4: For $k=k_{\min }$ to $k_{\max }$

(a) Evaluate the DTW distance for all pairs of generators $(p, q)$ of the clusters, and form the normalized dissimilarity matrix $\boldsymbol{d}_{\boldsymbol{d} t \boldsymbol{w}}^{*}$ of size $c$ x $c$, where $c$ is the number of clusters formed. (b) Merge the generator clusters with the smallest dissimilarity (suppose that they are $G_{p}$ and $G_{q}$ ) into a new cluster $G_{r}$, and reduce the value of $c$ by 1 (i.e., $\left.c_{n}=c_{n-1}-1\right)$.

(c) Check the current number of clusters, $c$. If this number is greater than the desired number of clusters, $k$, go to (a); otherwise, proceed to (d).

(d) Evaluate the Hubert-Levin index (C-index) and store it in matrix $\boldsymbol{H L}$.

Step 5: Find the minimum value of the matrix $\boldsymbol{H} \boldsymbol{L}$ which also corresponds to the optimal number of clusters.

Step 6: Output the optimal number of clusters, the $\boldsymbol{H} \boldsymbol{L}$ index, and clustering results (i.e., groups of generators)

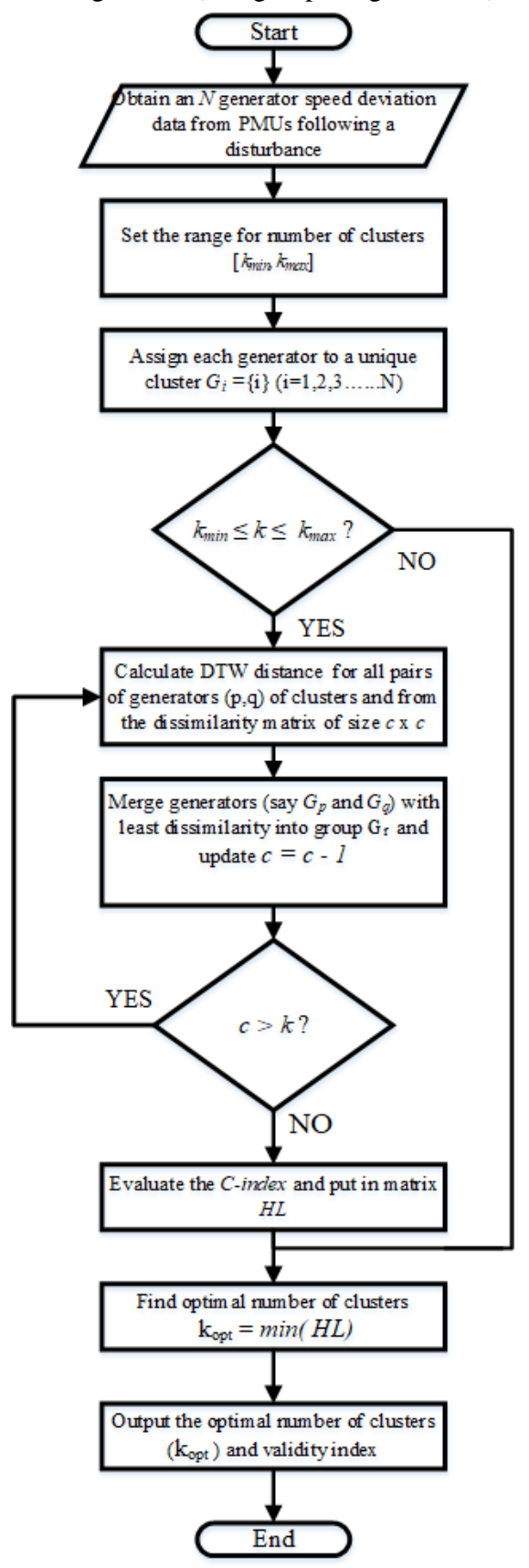

Figure 5. Flowchart of the coherency identification algorithm 


\section{TEST SYSTEM, SIMULATION RESULTS, AND PERFORMANCE EVALUATION}

The scheme was validated through dynamic simulations of the IEEE 39-bus test system. The system, also known as the New England test system, is a standard system widely used for transient stability studies, including coherency detection [1]. It is shown as Figure 6. It consists of 10 generators and 39 buses. Generator 1 (G1) is a generator representing a large system. Data for the modeling of the test system was obtained from [29].

The modeling and simulation of the test system were carried out using the Power System Simulator for Engineers (PSSE) software [30]. Cascading outage scenarios were created by creating three-phase faults at various buses and on various lines, and the resulting data used to test the proposed scheme. The proposed coherency algorithm was run on MATLAB ${ }^{\circledR}$ software.

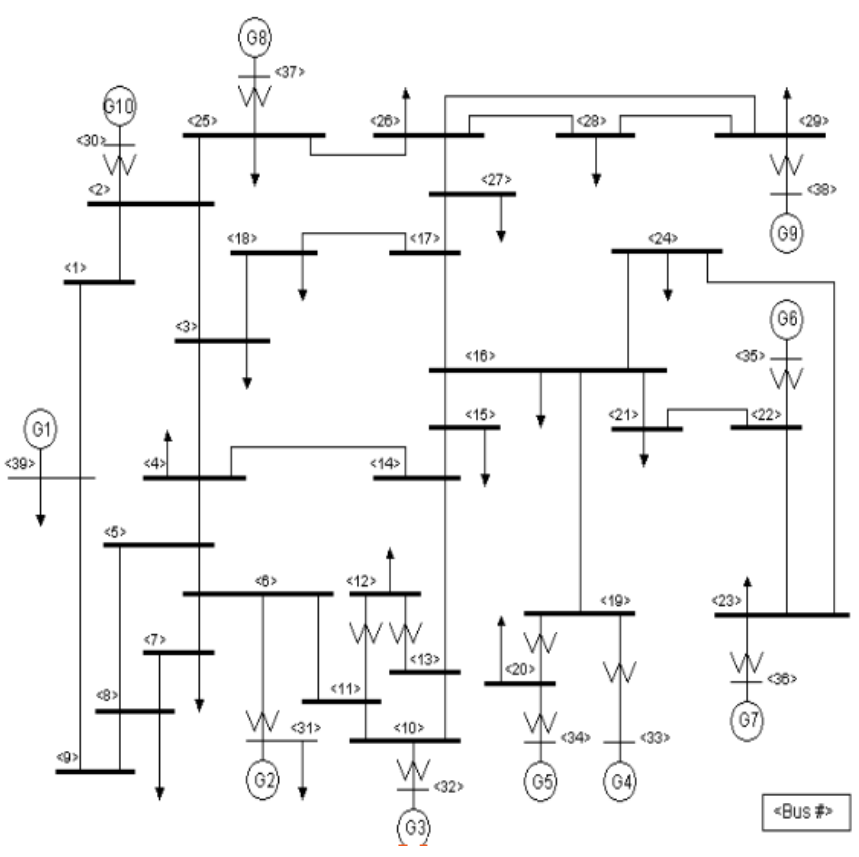

Figure 6. IEEE 39-bus test system

\section{Validation Results of Proposed Scheme}

Post-fault data corresponding to a time window of $0.5 \mathrm{~s}$ was chosen in order to accurately detect the consistency in the changes in the trajectories of the input parameter. Speed deviation data from 35 fault events were used to test the proposed scheme.

A sample performance of the proposed method is presented for a three-phase fault on bus 32 with a base load condition. For this case, the fault was applied at time $t_{1}=0.2 \mathrm{~s}$ and cleared at time $t_{2}=1.2 \mathrm{~s}$, resulting in some generators going out of step. Figure 7 shows a plot of the speed deviation data for the event. It can be observed from the figure that generators 2 and 3 (G2 and G3) form one coherent group while generators $4,5,6,7,8$, and 9 (G4, G5, G6, G7, G8, and G9) form another coherent group, with Generators 1 and 10 (G1 and G10) being singleton clusters. The data from the same fault were used to simulate the case of inconsistent delay times. For example, the data from G4 was delayed by $60 \mathrm{~ms}$ to simulate a real-time relative transmission delay of $60 \mathrm{~ms}$.
The normalized DTW dissimilarity matrix for this fault for consistent transmission delay times is as shown in Table 1. It can be noted that the dissimilarity values between generators in the same coherent group are small while those that are not coherent have high values. For example, for the third coherent group (G4, G5, G6, G7, G8, G9), the DTW dissimilarity value is approximately 0.05 . Also, the dissimilarity between G2 and G3, which also form one coherent group, is 0.1 . However, the dissimilarity value between G2 and G10 which are not coherent is 1.0. The DTW dissimilarity matrix for the $60 \mathrm{~ms}$ relative delay of data for G4 is as shown in Table 2. It can be observed that there are changes in the dissimilarity values of the group to which G4 belongs. For example, the new dissimilarity value between G4 and G8 is 0.1 as compared to the previous value (value in Table 1) of 0.04. However, there was no significant change in dissimilarity value between G4 and other non-coherent generators.

Table 3 shows the C-index evaluated for various number of clusters for both consistent and inconsistent delay cases. It can be observed that for a cluster search range of [2,7], the cluster number $K=4$ has the least index, indicating the optimal number of clusters is 4 . The coherent groups thus identified are as shown in Table 4. The results indicate that, the scheme can accurately identify the coherent groups for both consistent and inconsistent data transmission delay times. Similar responses were obtained for the other 34 fault cases.

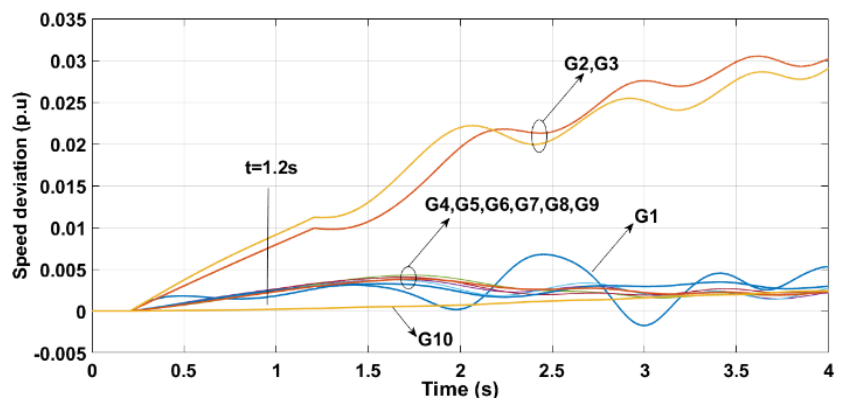

Figure 7. Rotor speed deviations for a three-phase fault on Bus 32

Table 1. Dissimilarity matrix for a three-phase fault on Bus 32 for consistent transmission delay

\begin{tabular}{lcccccccccc}
\hline \multicolumn{10}{c}{ DTW } \\
\hline & G1 & G2 & G3 & G4 & G5 & G6 & G7 & G8 & G9 & G10 \\
\hline G1 & $\mathbf{0 . 0}$ & 0.8 & 0.8 & 0.4 & 0.4 & 0.5 & 0.44 & 0.45 & 0.43 & 0.5 \\
& & & & & & & & & & \\
\hline G2 & 0.8 & $\mathbf{0 . 0}$ & $\mathbf{0 . 1}$ & 0.7 & 0.6 & 0.7 & 0.71 & 0.73 & 0.72 & 1.0 \\
& & & & & & & & & & \\
\hline G3 & 0.8 & $\mathbf{0 . 1}$ & $\mathbf{0 . 0}$ & 0.8 & 0.67 & 0.7 & 0.75 & 0.74 & 0.75 & 0.94 \\
\hline G4 & 0.4 & 0.7 & 0.8 & $\mathbf{0 . 0}$ & $\mathbf{0 . 0 4}$ & $\mathbf{0 . 0 5}$ & $\mathbf{0 . 0 3}$ & $\mathbf{0 . 0 4}$ & $\mathbf{0 . 0 4}$ & 0.4 \\
& & & & & & & & & & \\
\hline G5 & 0.4 & 0.6 & 0.67 & $\mathbf{0 . 0 4}$ & $\mathbf{0 . 0}$ & $\mathbf{0 . 0 4}$ & $\mathbf{0 . 0 5}$ & $\mathbf{0 . 0 4}$ & $\mathbf{0 . 0 5}$ & 0.4 \\
& & & & & & & & & & \\
\hline G6 & 0.5 & 0.7 & 0.7 & $\mathbf{0 . 0 5}$ & $\mathbf{0 . 0 4}$ & $\mathbf{0 . 0}$ & $\mathbf{0 . 0 6}$ & $\mathbf{0 . 0 5}$ & $\mathbf{0 . 0 5}$ & 0.42 \\
& & & & & & & & & & \\
\hline G7 & 0.44 & 0.71 & 0.75 & $\mathbf{0 . 0 3}$ & $\mathbf{0 . 0 5}$ & $\mathbf{0 . 0 6}$ & $\mathbf{0 . 0}$ & $\mathbf{0 . 0 5}$ & $\mathbf{0 . 0 6}$ & 0.41 \\
& & & & & & & & & & \\
\hline G8 & 0.45 & 0.73 & 0.74 & $\mathbf{0 . 0 4}$ & $\mathbf{0 . 0 4}$ & $\mathbf{0 . 0 5}$ & $\mathbf{0 . 0 5}$ & $\mathbf{0 . 0}$ & $\mathbf{0 . 0 6}$ & 0.4 \\
\hline G9 & 0.43 & 0.72 & 0.75 & $\mathbf{0 . 0 4}$ & $\mathbf{0 . 0 5}$ & $\mathbf{0 . 0 5}$ & $\mathbf{0 . 0 6}$ & $\mathbf{0 . 0 6}$ & $\mathbf{0 . 0}$ & 0.45 \\
& & & & & & & & & & \\
\hline G10 & 0.5 & 1.0 & 0.94 & 0.4 & 0.4 & 0.42 & 0.41 & 0.4 & 0.45 & $\mathbf{0 . 0}$
\end{tabular}


Table 2. Dissimilarity matrix for a three-phase fault on Bus 32 for $60 \mathrm{~ms}$ transmission delay of G4 data

\begin{tabular}{lcccccccccc}
\hline \multicolumn{10}{c}{ DTW } \\
\hline & G1 & G2 & G3 & G4 & G5 & G6 & G7 & G8 & G9 & G10 \\
\hline G1 & 0.0 & 0.8 & 0.8 & $\mathbf{0 . 5 5}$ & 0.4 & 0.5 & 0.44 & 0.45 & 0.43 & 0.5 \\
& & & & & & & & & & \\
\hline G2 & 0.8 & 0.0 & 0.1 & $\mathbf{0 . 7 5}$ & 0.6 & 0.7 & 0.71 & 0.73 & 0.72 & 1.0 \\
& & & & & & & & & & \\
\hline G3 & 0.8 & 0.1 & 0.0 & $\mathbf{0 . 8 3}$ & 0.67 & 0.7 & 0.75 & 0.74 & 0.75 & 0.94 \\
\hline G4 & $\mathbf{0 . 5 5}$ & $\mathbf{0 . 7 5}$ & $\mathbf{0 . 8 3}$ & $\mathbf{0 . 0}$ & $\mathbf{0 . 1}$ & $\mathbf{0 . 1 2}$ & $\mathbf{0 . 0 9}$ & $\mathbf{0 . 1}$ & $\mathbf{0 . 1 1}$ & $\mathbf{0 . 4 5}$ \\
\hline G5 & 0.4 & 0.6 & 0.67 & $\mathbf{0 . 1}$ & 0.0 & 0.04 & 0.05 & 0.04 & 0.05 & 0.4 \\
& & & & & & & & & & \\
\hline G6 & 0.5 & 0.7 & 0.7 & $\mathbf{0 . 1 2}$ & 0.04 & 0.0 & 0.06 & 0.05 & 0.05 & 0.42 \\
\hline G7 & 0.44 & 0.71 & 0.75 & $\mathbf{0 . 0 9}$ & 0.05 & 0.06 & 0.0 & 0.05 & 0.06 & 0.41 \\
& & & & & & & & & & \\
\hline G8 & 0.45 & 0.73 & 0.74 & $\mathbf{0 . 1}$ & 0.04 & 0.05 & 0.05 & 0.0 & 0.06 & 0.4 \\
\hline G9 & 0.43 & 0.72 & 0.75 & $\mathbf{0 . 1 1}$ & 0.05 & 0.05 & 0.06 & 0.06 & 0.0 & 0.45 \\
\hline G10 & 0.5 & 1.0 & 0.94 & $\mathbf{0 . 4 5}$ & 0.4 & 0.42 & 0.41 & 0.4 & 0.45 & $\mathbf{0 . 0}$ \\
\end{tabular}

Table 3. C-index of different numbers of clusters for a fault on

\begin{tabular}{lcccccc}
\multicolumn{7}{c}{ Bus 32} \\
\hline \multicolumn{1}{c}{$\mathbf{K}$} & $\mathbf{2}$ & $\mathbf{3}$ & $\mathbf{4}$ & $\mathbf{5}$ & $\mathbf{6}$ & $\mathbf{7}$ \\
\hline $\begin{array}{l}\text { C-index } \\
\text { for } \\
\text { consistent } \\
\text { delay }\end{array}$ & 0.68 & 0.53 & $\mathbf{0 . 4 2}$ & 0.64 & 0.76 & 0.82 \\
\hline $\begin{array}{l}\text { C-index } \\
\text { for } 60 \mathrm{~ms} \\
\text { delay of }\end{array}$ & 0.81 & 0.61 & $\mathbf{0 . 5 1}$ & 0.72 & 0.84 & 0.95 \\
G4 data & & & & & & \\
& & & & & & \\
& & & & & & \\
\end{tabular}

Table 4. Formed coherent groups for a fault on Bus 32

\begin{tabular}{lc}
\hline \multicolumn{1}{c}{ Type of event } & Clustering results \\
\hline Three-phase short circuit at bus 32 & $(1),(2,3)$ \\
& $(4,5,6,7,8,9),(10)$ \\
\hline Three-phase short circuit at bus 32 & $(1),(2,3)$ \\
with 60 ms relative delay of PMU data & $(4,5,6,7,8,9),(10)$ \\
from G4 &
\end{tabular}

\section{DTW distance as an Index for Characterizing PMU data Transmission Delay of a WAMS}

The DTW distance is a function of the PMU data transmission delay and could be used as an index for characterizing the transmission delay of WAMS. This was validated by using data from 10 of the 35 three-phase fault simulations. A typical case is the fault on bus 32 as indicated earlier.

In this analysis, two coherent generators (G4 and G6) were selected, and their post-fault time-domain variations observed as shown in Figure 8(a). The speed deviation data of G6 were then delayed relative to $\mathrm{G} 4$ in time steps of $20 \mathrm{~ms}$ from 0 s to $120 \mathrm{~ms}$. A time-delay of $0 \mathrm{~s}$ means the two trajectories are timesynchronized. The normalized DTW distance for each time delay is computed and plotted against the relative delay time as shown in Figure 8(b). It can be observed from Figure 8(b) that the DTW distance between the two signals is proportional to the relative delay time.
Thus, the DTW distance in addition to being used as a measure of coherency, can be used as an index to the transmission delay time experienced by a particular signal in WAMS. In other words, given a DTW distance, one can have an idea of the delay time of a system. The higher the delay time, the higher the DTW distance and vice versa.

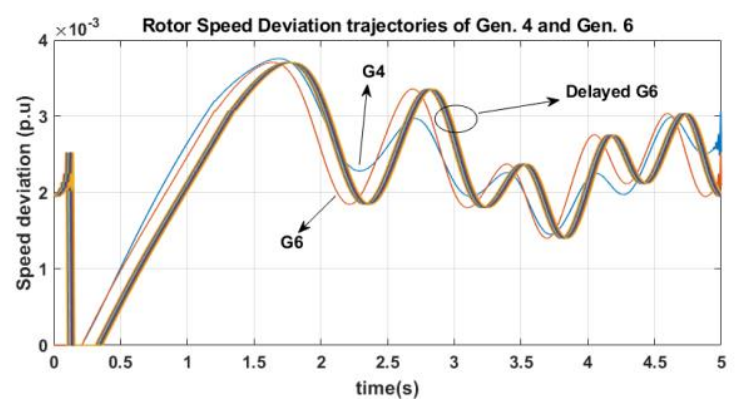

(a)

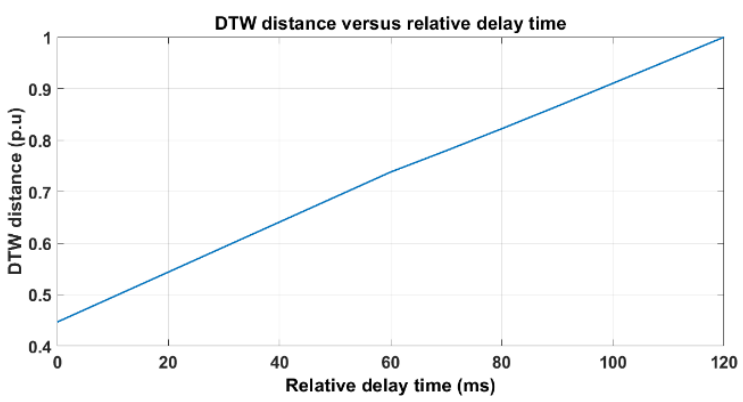

(b)

Figure 8. (a) Speed deviation trajectories of generators 4 and 6 (b) DTW distance versus relative delay time

\section{Validation of Data Delay Factor}

The data delay factor was validated by using 15 of the 35 fault cases. One is the three-phase fault on Bus 32. This resulted in four clusters as earlier indicated in Figure 7. The validation was done by assuming three instances of data transmission delay. The first instance is when there are consistent transmission delay times, simulated by using the original data obtained from PSSE. The second and third cases involve the same fault event, however, the speed deviation data of G6 was intentionally delayed by 20 $\mathrm{ms}$ and $60 \mathrm{~ms}$ respectively with respect to the other generators. The data delay factor matrices for the three instances are shown in Tables 5, 6 and 7. Only the upper diagonals are shown because the matrices are symmetrical.

Table 5 shows the delay factor data for the case where there are consistent transmission delay times. It can be observed that the delay factors for all pairs of generators are approximately unity. This confirms the fact that for consistent transmission delays, the Euclidean distance $\left(d_{\text {euc }}\right)$ closely tracks the DTW distance $\left(d_{d t w}\right)$ for both coherent and non-coherent signal pairs as indicated earlier.

Table 6 contains delay factors of pairs of generators for the same fault but for a relative delay of $20 \mathrm{~ms}$ for speed deviation data of G6. It can be observed from the matrix that the delay factors between G6 and generators belonging to other coherent groups, i.e., G1, G2, G3, and G10 are close to unity. However, the delay factors between G6 and generators in the same coherent group 
deviate largely from unity. For example, the delay factor between G6 and G8 belonging to the same cluster is 0.52 , as opposed to the consistent transmission delays scenario value of 0.92 in Table 5. For the matrix in Table 7 corresponding to the relative delay of speed deviation data of G6 of $60 \mathrm{~ms}$, it can be noted that the delay factors between G6 and associated coherent generators further deviate from unity. For instance, the delay factor between G6 and G8 is 0.14 as opposed to 0.52 for the $20 \mathrm{~ms}$-delay scenario. These also confirm the fact that for inconsistent transmission delay times, the Euclidean distance $\left(d_{\text {euc }}\right)$ tracks the DTW distance $\left(d_{d t w}\right)$ for non-coherent signal pairs. However, for coherent signal pairs, the DTW distance deviates from the Euclidean distance, and this deviation is proportional to the relative delay time.

Thus, the data delay factor can be used to determine, for a given disturbance, whether there is a relative transmission delay of a signal, and the generator(s) whose signal(s) is (are) experiencing such delay(s). This feature will further be explored in a future work to determine the necessary control signal to apply to a phasor data concentrator in the data transmission path.

Table 5. Delay factor matrix for a fault on Bus 32 for consistent delay times.

\begin{tabular}{|c|c|c|c|c|c|c|c|c|c|}
\hline \multicolumn{10}{|c|}{$\mathbf{d}_{\mathrm{dtw}} / \mathrm{d}_{\mathrm{euc}}$} \\
\hline G1 & G2 & G3 & G4 & G5 & G6 & G7 & G8 & G9 & G10 \\
\hline G1 & 0.84 & 0.84 & 0.86 & 0.87 & 0.8 & 0.79 & 0.84 & 0.94 & 0.94 \\
\hline G2 & & 0.89 & 0.84 & 0.88 & 0.81 & 1.01 & 0.7 & 0.98 & 1.01 \\
\hline G3 & & & 0.8 & 0.79 & 0.82 & 1.02 & 1.01 & 0.92 & 1.04 \\
\hline G4 & & & & 0.91 & 0.90 & 0.93 & 0.95 & 0.91 & 0.95 \\
\hline G5 & & & & & 0.92 & 0.95 & 0.96 & 0.92 & 0.93 \\
\hline G6 & & & & & & 1.01 & 0.92 & 0.89 & 0.96 \\
\hline G7 & & & & & & & 0.92 & 0.91 & 0.93 \\
\hline G8 & & & & & & & & 0.92 & 0.97 \\
\hline G9 & & & & & & & & & 0.92 \\
\hline
\end{tabular}

Table 6. Delay factor matrix for a fault on Bus 32, with 20msdelay of speed deviation data of Generator 6 .

\begin{tabular}{|c|c|c|c|c|c|c|c|c|c|c|}
\hline \multicolumn{11}{|c|}{$\mathbf{d}_{\mathrm{dtw}} / \mathbf{d}_{\mathrm{euc}}$} \\
\hline & G1 & G2 & G3 & G4 & G5 & G6 & G7 & G8 & G9 & G10 \\
\hline G1 & & 0.84 & 0.84 & 0.86 & 0.87 & 1.1 & 0.79 & 0.84 & 0.94 & 0.94 \\
\hline G2 & & & 0.89 & 0.84 & 0.88 & 0.97 & 1.01 & 0.7 & 0.98 & 1.01 \\
\hline G3 & & & & 0.8 & 0.79 & 0.82 & 1.02 & 1.01 & 0.92 & 1.04 \\
\hline G4 & & & & & 0.91 & 0.5 & 0.93 & 0.95 & 0.91 & 0.95 \\
\hline G5 & & & & & & 0.52 & 0.95 & 0.96 & 0.92 & 0.93 \\
\hline G6 & & & & & & & 0.53 & 0.52 & 0.6 & 1.04 \\
\hline G7 & & & & & & & & 0.92 & 0.91 & 0.93 \\
\hline G8 & & & & & & & & & 0.92 & 0.97 \\
\hline G9 & & & & & & & & & & 0.92 \\
\hline
\end{tabular}

Table 7. Delay factor matrix for a fault on Bus 32, with 60msdelay of speed deviation data of Generator 6.

\begin{tabular}{lcccccccccc}
\hline \multicolumn{10}{c}{ delay of speed deviation data of Generator 6. } \\
\hline & G1 & G2 & G3 & G4 & G5 & G6 & G7 & G8 & G9 & G10 \\
& & & & & & & & & \\
\hline G1 & 0.84 & 0.84 & 0.86 & 0.87 & 0.8 & 0.79 & 0.84 & 0.94 & 0.94
\end{tabular}

\begin{tabular}{|c|c|c|c|c|c|c|c|c|}
\hline G2 & 0.89 & 0.84 & 0.88 & 0.81 & 1.01 & 0.7 & 0.98 & 1.01 \\
\hline G3 & & 0.8 & 0.79 & 0.82 & 1.02 & 1.01 & 0.92 & 1.04 \\
\hline G4 & & & 0.91 & 0.15 & 0.93 & 0.95 & 0.91 & 0.95 \\
\hline G5 & & & & 0.18 & 0.95 & 0.96 & 0.92 & 0.93 \\
\hline G6 & & & & & 0.21 & 0.14 & 0.12 & 0.84 \\
\hline G7 & & & & & & 0.92 & 0.91 & 0.93 \\
\hline G8 & & & & & & & 0.92 & 0.97 \\
\hline G9 & & & & & & & & 0.92 \\
\hline
\end{tabular}

\section{CONCLUSIONS}

A method to determine coherent clusters of synchronous generators in power systems based on windowed dynamic time warping has been presented. It is an online method. The method uses rotor speed deviation as input parameter. Rotor deviations can be captured using phasor measurement units which makes the method feasible for practical implementation. The major merit of the technique is its ability to accurately identify coherent clusters in the event of inconsistent data delay or data loss. The method is accurate and can effectively aid intentional controlled islanding.

\section{REFERENCES}

[1] E. Frimpong, J. Asumadu, and P. Okyere, "Real time prediction of coherent generator groups," Journal of Electrical Engineering, vol. 16, pp. 47-56, 2016. Available:http://jee.ro/covers/art.php?issue=WD1448 649431W5658a2d7c8a7d

[2] Y. Chen, Z. Zhang, X. Song, J. Liu, M. Hou, G. Li, W. $\mathrm{Xu}$, and J. Ma, "Coherent Clustering Method Based on Weighted Clustering of Multi-Indicator Panel Data," IEEE Access, vol. 7, pp. 43462-43472, 2019.Available:https://ieeexplore.ieee.org/document/ 8673899

[3] A. R. Hassani Ahangar, G. B. Gharehpetian, and H. R. Baghaee, "A review on intentional controlled islanding in smart power systems and generalized framework for ICI in microgrids," International Journal of Electrical Power \& Energy Systems, vol. 118, 2020. Available:

http://www.sciencedirect.com/science/article/pii/S01 42061519314474

[4] H. U. Banna, Z. Yu, D. Shi, Z. Wang, D. Su, C. Xu, S. K. Solanki, and J. M. Solanki, "Online coherence identification using dynamic time warping for controlled islanding," Journal of Modern Power Systems and Clean Energy, vol. 7, no. 1, pp. 38-54, 2019. Available: https://doi.org/10.1007/s40565-018-0443-z

[5] O. Gomez and M. A. Rios, "Real time identification of coherent groups for controlled islanding based on graph theory," IET Generation, Transmission \& Distribution, vol. 9, no. 8, pp. 748-758, 2015. Available: https://digitallibrary.theiet.org/content/journals/10.1049/ietgtd.2014.0865?crawler $=$ redirect\&mimetype $=$ applicat ion/pdf 
[6] Y. Ota, H. Ukai, K. Nakamura, and H. Fujita, "PMU based midterm stability evaluation of wide-area power system," in IEEE/PES Transmission and Distribution Conference and Exhibition, vol. 3, pp. 1676-1680 vol.3, 2002

[7] M. Jonsson, M. Begovic, and J. Daalder, "A new method suitable for real-time generator coherency determination," IEEE Transactions on Power Systems, vol. 19, no. 3, pp. 1473-1482, 2004. Available: https://ieeexplore.ieee.org/document/1318684

[8] S. A. Siddiqui, K. Verma, K. R. Niazi, and M. Fozdar, "Real-Time Monitoring of Post-Fault Scenario for Determining Generator Coherency and Transient Stability Through ANN," IEEE Transactions on Industry Applications, vol. 54, no. 1, pp. 685-692, 2018.

[9] M. A. A. Abd-EI-Rehim, I. D. Helal, and M. A. H. Omar, "Multi-machine power system dynamic equivalents using artificial intelligence (ANN)," in 2006 Eleventh International Middle East Power Systems Conference, vol. 1, pp. 197-207, 2006,.

[10]A. Vahidnia, G. Ledwich, E. Palmer, and A. Ghosh, "Generator coherency and area detection in large power systems," Generation, Transmission \& Distribution, IET, vol. 6, pp. 874-883, 2012.

[11]F. Hashiesh, H. E. Mostafa, I. Helal and M. M. Mansour, "Determination of generators coherent groups based on synchrophasors using bioinformatics toolbox," IEEE PES Conference on Innovative Smart Grid Technologies - Middle East, pp. 1-7, 2011. Available:

https://ieeexplore.ieee.org/document/6220819

[12] M. Davodi, H. R. Modares, E. Reihani, and A. Sarikhani, "Coherency Approach by Hybrid PSO, KMeans Clustering Method in Power System," in 2008 IEEE 2nd International Power and Energy Conference, Johor Bahru, Malaysia, vol. 63, pp. 1203 1207, 2009.

[13] K. K. Anaparthi, B. Chaudhuri, N. F. Thornhill, and B. C. Pal, "Coherency identification in power systems through principal component analysis," IEEE Transactions on Power Systems, vol. 20, no. 3, pp. 1658-1660, 2005. Available: https://ieeexplore.ieee.org/document/1490624

[14]M. A. Mohd Ariff and B. C. Pal, "Coherency Identification in Interconnected Power System-An Independent Component Analysis Approach," Power Systems, IEEE Transactions on, vol. 28, pp. 17471755, 2013. Available: https://ieeexplore.ieee.org/document/6331591

[15] S. Avdaković, E. Bećirović, A. Nuhanović, and M. Kušljugić, "Generator Coherency Using the Wavelet Phase Difference Approach," IEEE Transactions on Power Systems, vol. 29, no. 1, pp. 271-278, 2014. Available: https://ieeexplore.ieee.org/document/6595731

[16] W. Yao, D. Zhou, L. Zhan, Y. Liu, Y. Cui, S. You, and Y. Liu, "GPS signal loss in the wide area monitoring system: Prevalence, impact, and solution," Electric Power Systems Research, vol. 147, pp. 254-262, 2017. Available:

http://www.sciencedirect.com/science/article/pii/S03 78779617300950
[17] C. Cassisi, P. Montalto, M. Aliotta, A. Cannata, and A. Pulvirenti, "Similarity Measures and Dimensionality Reduction Techniques for Time Series Data Mining," in Advances in Data Mining Knowledge Discovery and Applications, A. Karahoca, Ed.: IntechOpen, 2012.

[18] S. Zhou, Z. Xu, and F. Liu, "Method for Determining the Optimal Number of Clusters Based on Agglomerative Hierarchical Clustering," in IEEE Transactions on Neural Networks and Learning Systems, vol. 28, no. 12, pp. 3007-3017, 2017. Available: https://ieeexplore.ieee.org/document/7583663

[19] E. Dimitriadou, S. Dolničar, and A. Weingessel, "An examination of indexes for determining the number of clusters in binary data sets," Psychometrika, vol. 67, no. 1 , pp. 137-159, 2002. Available: https://doi.org/10.1007/BF02294713

[20] M. Chenine and L. Nordström, "Investigation of communication delays and data incompleteness in multi-PMU Wide Area Monitoring and Control Systems," presented at the 2009 International Conference on Electric Power and Energy Conversion Systems, (EPECS), pp. 1-6, 2009. Available: https://ieeexplore.ieee.org/document/5415702?arnum ber $=5415702$.

[21] S. Grewal, M. K. Soni, and D. K. Jain, "Requirements and challenges of PMUs communication in WAMS environment," Far East Journal of Electronics and Communications, vol. 13, pp. 121-135, 12/01 2014. Available:

https://www.researchgate.net/publication/282751447

_Requirements_and_challenges_of_PMUs_communi cation_in_WAMS_environment

[22]Z. Lin, F. Wen, Y. Ding, Y. Xue, S. Liu, Y. Zhao, and S. Yi, "WAMS-Based Coherency Detection for Situational Awareness in Power Systems With Renewables," IEEE Transactions on Power Systems, vol. 33, no. 5, pp. 5410-5426, 2018. Available: https://ieeexplore.ieee.org/document/8326526

[23] V. Siyoi, S. Kariuki, and M. Saulo, "Strategic PMU placement for stability enhancement," International Journal of Energy and Power Engineering. Special Issue: Electrical Power Systems Operation and Planning, vol. 4, pp. 81-94, 2015. Available: http://www.sciencepublishinggroup.com/journal/pape rinfo? journalid $=164 \&$ doi $=10.11648 /$ j.ijepe.s. 2015040 201.18

[24]A. Abdelaziz, A. Ibrahim, and Z. Hasan, "Phasor measurement units for out of step detection," The International Conference on Electrical Engineering, vol. 8, pp. 1-19, 2012. Available: https://iceeng.journals.ekb.eg/article_30796.html

[25] I. Davidson and S. S. Ravi, "Agglomerative hierarchical clustering with constraints: Theoretical and empirical results," in European Conference on Principles of Data Mining and Knowledge Discovery, pp. 59-70: Springer, 2005.

[26] M. Charrad, N. Ghazzali, V. Boiteau, and A. Niknafs, "An examination of indices for determining the number of clusters : NbClust Package," ed, 2013

[27]E. Keogh and C. A. Ratanamahatana, "Exact indexing of dynamic time warping," Knowledge and 
Information Systems, vol. 7, no. 3, pp. 358-386, 2005/03/01 2005. Available: https://doi.org/10.1007/s10115-004-0154-9

[28] Y. Chen, B. Hu, E. Keogh, and G. Batista, "DTW-D: time series semi-supervised learning from a single example," in Proceedings of the 19th ACM SIGKDD international conference on Knowledge discovery and data mining, pp. 383-391. 2013. Available: https://dl.acm.org/doi/abs/10.1145/2487575.2487633

[29] S. Y, "Design of Secondary Voltage and Stability Controls with Multiple Control Objectives," PhD Dissertation, Georgia Institute of Technology, Georgia, USA, 2009. Available: https://citeseerx.ist.psu.edu/viewdoc/download?doi=1 0.1.1.590.3831\&rep $=$ rep1\&type $=$ pdf

[30]"Power System Simulator for Engineers, PSSE University Edition. Available: https://new.siemens.com/global/en/products/energy/e nergy-automation-and-smart-grid/pss-software/pssexplore-order-form.html

\section{NOMENCLATURE}

$\delta \quad$ speed deviation threshold

$\varepsilon \quad$ warping window threshold

$\gamma \quad$ cumulative distance matrix

$\omega \quad$ speed deviation, $\mathrm{rad} / \mathrm{s}$ 